\title{
Endoscopy-verified occult subependymal dissemination of glioblastoma and brain metastasis undetected by MRI: prognostic significance
}

This article was published in the following Dove Press journal:

OncoTargets and Therapy

12 December 2012

Number of times this article has been viewed

\author{
Maurizio lacoangeli' \\ Alessandro Di Rienzo' \\ Roberto Colasanti' \\ Antonio Zizzi ${ }^{2}$ \\ Maurizio Gladi' \\ Lorenzo Alvaro' \\ Niccolò Nocchi' \\ Lucia Giovanna Maria \\ Di Somma' \\ Marina Scarpelli² \\ Massimo Scerrati' \\ 'Department of Neurosurgery, \\ ${ }^{2}$ Department of Pathology, Università \\ Politecnica delle Marche, Umberto I \\ General Hospital, Ancona, Italy
}

\begin{abstract}
Although various prognostic indices exist for patients with malignant brain tumors, the prognostic significance of the subependymal spread of intracranial tumors is still a matter of debate. In this paper, we report the cases of two intraventricular lesions, a recurrent glioblastoma multiforme (GBM) and a brain metastasis, each successfully treated with a neuroendoscopic approach. Thanks to this minimally invasive approach, we achieved good therapeutic results: we obtained a histological diagnosis; we controlled intracranial hypertension by treating the associated hydrocephalus and, above all, compared with a microsurgical approach, we reduced the risks related to dissection and brain retraction. Moreover, in both cases, neuroendoscopy enabled us to identify an initial, precocious subependymal tumor spreading below the threshold of magnetic resonance imaging (MRI) detection. This finding, undetected in pre-operative MRI scans, was then evident during follow-up neuroimaging studies. In light of these data, a neuroendoscopic approach might play a leading role in better defining the prognosis and optimally tailored management protocols for GBM and brain metastasis.
\end{abstract}

Keywords: subependymal spreading, glioblastoma, brain metastasis, endoscopy, minimally invasive surgery, prognosis

\section{Introduction}

Malignant histological findings can be observed in approximately $13 \%$ of tumors that arise within the ventricular system of the brain. These intraventricular tumors include the glioblastoma multiforme (GBM) and metastases, which represent a unique therapeutic challenge because of their deep location and their proximity to eloquent brain structures. ${ }^{1}$ The prognostic significance of subependymal dissemination for intracranial tumors remains unclear, despite having various prognostic indices for patients with GBMs or brain metastases: patient age, tumor size, Karnofsky Performance Scale (KPS) score, tumor resection, adjuvant therapies, tumor location, isocitrate dehydrogenase (IDH) mutational status, the Radiation Therapy Oncology Group's (RTOG's) recursive partitioning analysis (RPA) class, and, for metastatic brain tumors, the number of brain metastases, primary tumor type and activity, and the presence of extracranial systemic metastases..$^{2-7}$ In this article, we focus on the spreading of massive, endoscopy-verified, intraventricular lesions (a recurrent GBM and a brain metastasis) situated in the subependymal region and choroid plexus. These lesions were previously undetected in magnetic resonance imaging (MRI) and positron emission tomography (PET) scan. Thus, a neuroendoscopic approach allowed us to safely reach these deep seated lesions, minimizing dissection and brain retraction and consequently reducing the risk of injury to neurovascular structures. This minimally invasive approach 
represents an effective treatment for intraventricular lesions, permitting the acquisition of a histological diagnosis (and thus enabling a specific subsequent adjuvant therapy). This approach also restores cerebrospinal fluid (CSF) dynamics and allows for the insertion of an intraventricular catheter for selective intratechal drug administration. ${ }^{8}$

\section{Materials and methods}

\section{Surgical endoscopic technique}

A surgical endoscopic technique was performed on two patients. While these patients were under general anesthesia - total intravenous anesthesia (TIVA) - a precoronal burr hole was drilled on the mid pupillary line and, after the opening of the dura mater, the arachnoid was coagulated for insertion of a rigid, zero-degree endoscope (Karl Storz, Tuttlingen, Germany) (4 $\mathrm{mm}$ in diameter) in the ventricular system. After removal of the lesions by suction/dissection technique, CSF pathways were further relieved, performing a ventriculocisternostomy of the third ventricle in the first patient and a septostomy in the second. With the first patient, an intraventricular catheter was inserted at the end of the procedure, connecting the catheter with an Ommaya reservoir for intrathecal chemotherapy.

\section{Histopathological analysis}

Routine hematoxylin and eosin staining was performed in both cases. The O6-methylguanine-DNA methyltransferase (MGMT) status of the glioblastoma was determined using methylation-specific polymerase chain reaction (PCR). ${ }^{9}$ In the second reported case, immunohistochemical staining was performed on formalin-fixed, paraffin-embedded sections, using antibodies against S100, cytokeratin 7 and 20, carcinoembryonic antigen (CEA), thyroid transcription factor 1 (TTF-1), and synaptophysin.

\section{Results}

\section{Case I}

A 69-year-old man was admitted to our department for reevaluation because of a tumor recurrence. One year before re-evaluation, he had initially presented with headache, memory loss, and left homonymous hemianopia. Brain MRI had revealed a large, heterogeneously enhancing tumor in the right temporal lobe, and the tumor had extended to the choroid plexus of the right lateral ventricle and to the temporal horn, infiltrating the hippocampus (Figure 1A). He had undergone a right temporoparietal craniotomy with a gross total resection of the tumor (Figure 1B). The histological diagnosis had been GBM. Methylation-specific
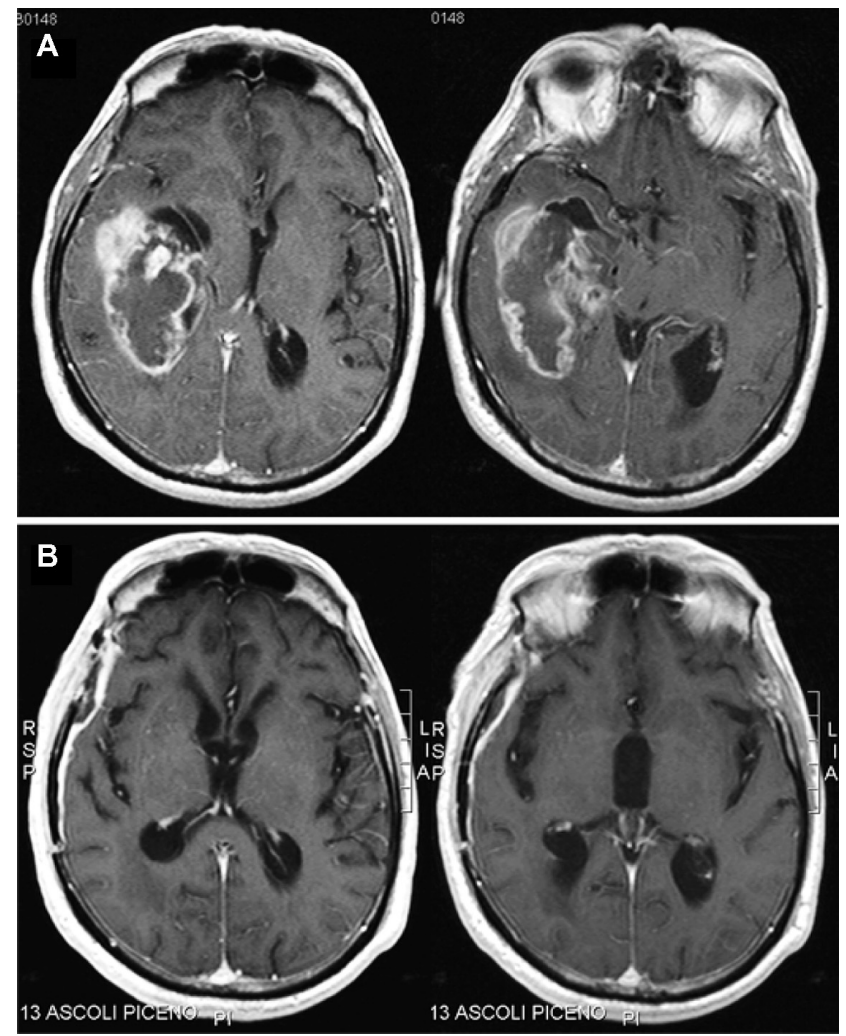

Figure I Contrast-enhanced, axial, TI-weighted magnetic resonance images of the tumor.

Notes: Initial images showing a large, heterogeneously enhancing tumor in the right temporal lobe, extending to the choroid plexus of the right lateral ventricle and to the temporal horn, infiltrating the hippocampus (A). Images at 6 months after the first operation, documenting a gross total resection of the tumor without evidence of recurrences $(\mathbf{B})$.

PCR analysis had shown a methylated MGMT promoter. The patient had an uneventful postoperative course and had received an adjuvant radiochemotherapy, according to the Stupp protocol. ${ }^{10}$ Brain MRI performed at 3 and 6 months after surgery had not shown tumor recurrences (Figure 1B). At an 11-month follow-up, a contrast enhanced MRI revealed the presence of an apparently solitary nodular lesion inside the third ventricle, while the operated site was still disease-free (Figure 2A).

Because of a high KPS score and the positive response to the integrated treatment of the original tumor site, we planned a minimally invasive endoscopic approach to remove the apparently solitary ventricular relapse. The patient underwent a purely neuroendoscopic procedure via a left precoronal transfrontal approach. The walls of the left lateral and third ventricles looked irregular and grayish. Neuroendoscopic examination, beyond the MRI-detected nodule inside the third ventricle, surprisingly revealed a diffuse tumor infiltration, composed of small adjacent nodules lying beneath the ependyma, unidentified by MRI and PET scan (Figure 3). We removed the biggest subependymal lesion inside the third 

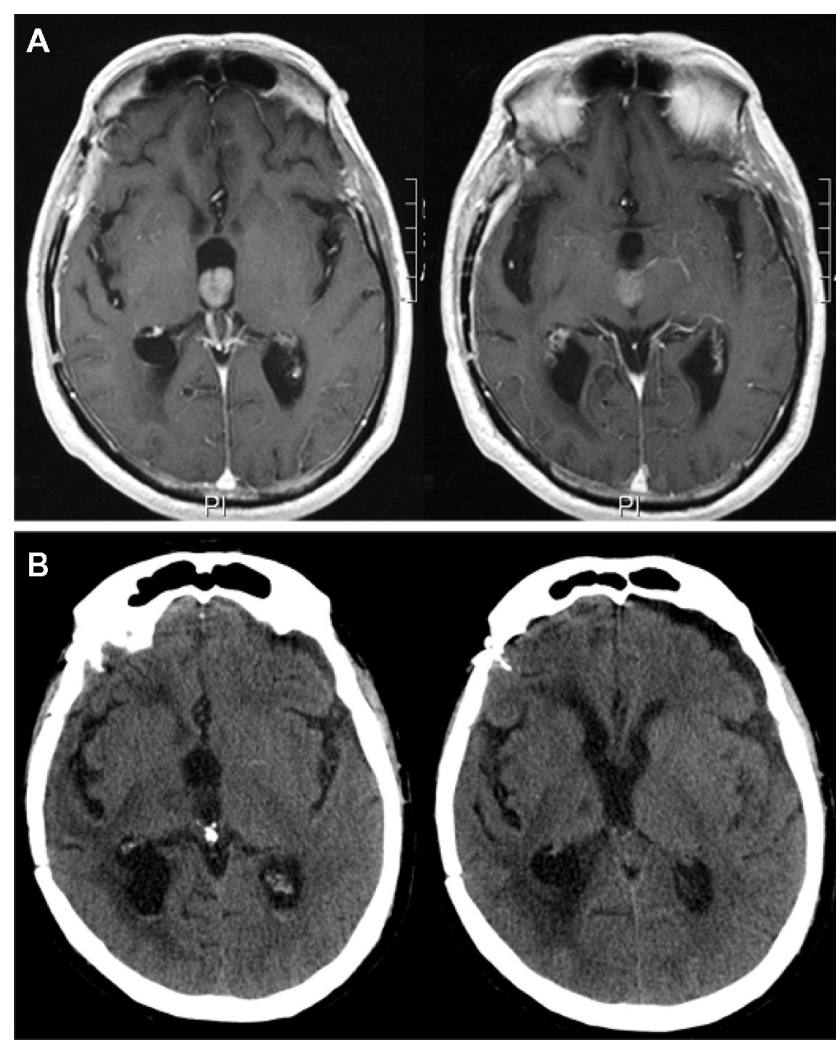

Figure 2 The patient's third ventricle nodular lesion.

Notes: Contrast-enhanced magnetic resonance images at the II-month follow-up revealing the onset of an apparently solitary nodular lesion in the third ventricle, while the operative site was still disease-free (A). Postoperative computed tomography scan showing the resection of the nodular lesion in the third ventricle without complications (B).

ventricle, performed a ventriculocisternotomy of the third ventricle, and placed an intraventricular catheter there, connecting the catheter to an Ommaya reservoir for locoregional intrathecal chemotherapy (Figure 2B). The histological diagnosis of GBM was confirmed (Figure 4). The postoperative course was uneventful, and the patient survived for 6 months after that surgical procedure.

\section{Case 2}

A 61-year-old woman presenting with an unsteady gait associated with headache and nausea was admitted to our department. Neurological examination revealed a 3-month history of mild memory disturbance and dizziness. Brain MRI at admission revealed multiple, heterogeneously enhancing lesions that were proximal to the choroid plexus of the right lateral ventricle, occurring with mild associated perilesional edema and right ventricular enlargement due to occlusion of the foramen of Monro. The left ventricle appeared normal (Figure 5). Magnetic resonance spectroscopy revealed an elevation in choline and a reduction in $\mathrm{N}$-acetyl aspartate in the area of the lesions, indicative of a

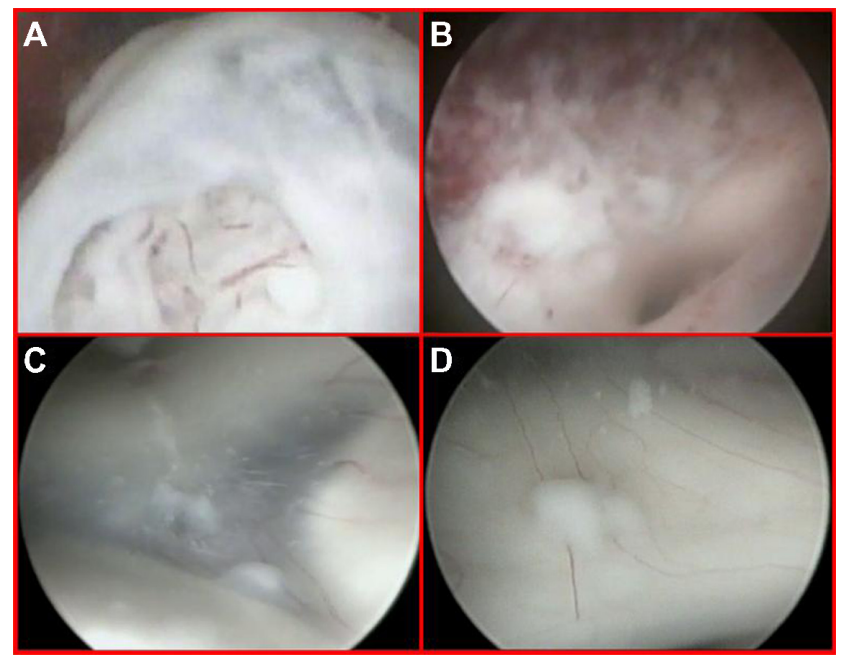

Figure 3 Close-up endoscopic third ventricle views.

Notes: The nodule inside the third ventricle, just beneath the opened ependymal layer (A). Endoscopic view after tumor removal with the origin of the sylvian aqueduct $(\mathbf{B})$. The floor of the third ventricle detecting a diffuse tumor infiltration composed of small adjacent nodules lying beneath the ependyma, unidentified by MRI and PET scan (C and D).

high-grade tumor. The patient, with unknown primary cancer in anamnesis, underwent a neuroendoscopic procedure for the resection of the lesion at the level of the foramen of Monro in order to obtain a diagnosis and to address the hydrocephalus (Figure 5B and E). A septostomy was performed for the same reason (Figure 6). Neuroendoscopic examination revealed a diffuse yellowish coloration of the walls of the right lateral ventricle with clusters of neoplastic cells embedding the choroid plexus. A thin sheet of tumor cells forming small nodules was present in the subependymal region. This infiltration showed a perivascular pattern and spread beneath the normal ependyma (Figure 6A-C). Interestingly, via the

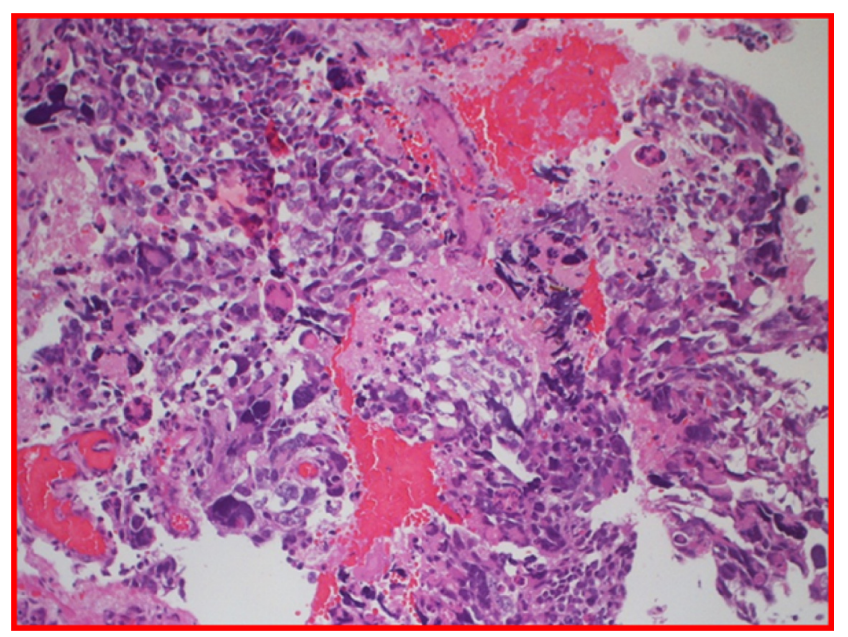

Figure $4 \mathrm{H}$ and $\mathrm{E}$ glioblastoma multiforme, World Health Organization grade IV, with evidence of nuclear pleomorphism, mitotic activity, endothelial hyperplasia, and areas of palisading necrosis. 


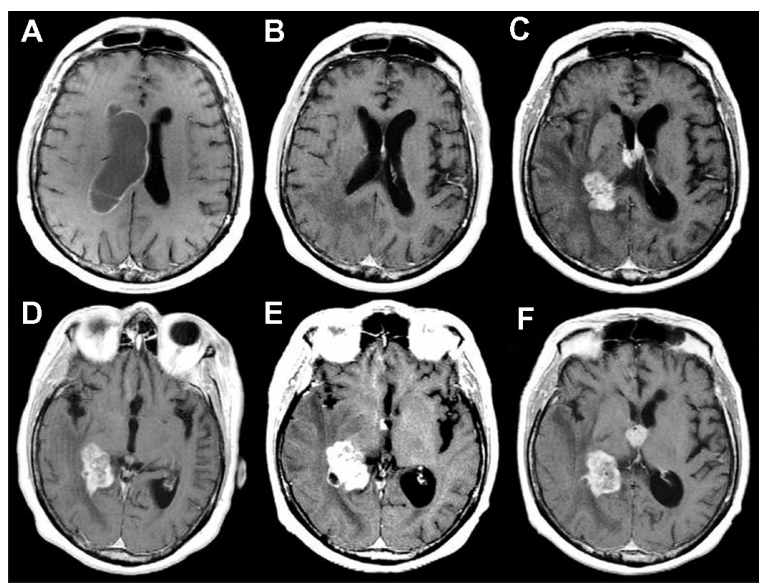

Figure 5 Preoperative, contrast-enhanced, axial, TI-weighted magnetic resonance images.

Notes: Multiple heterogeneously enhanced lesions, proximal to the choroid plexus of the right lateral ventricle, with mild associated perilesional edema and right ventricular enlargement due to occlusion of the foramen of Monro* (A and $\mathbf{D}$ ). Contrast-enhanced, axial, TI-weighted magnetic resonance image at the 6-month follow-up showing the resolution of the obstructive hydrocephalus (B). At this time, MRI also documented the contralateral tumor infiltration along the anterior commissure (E), not detected at the preoperative MRI (D), although it was evident during the endoscopic procedure. Contrast-enhanced, axial, TI-weighted MR image at the 18-month follow-up showing a massive subependymal tumor infiltration of the left lateral and third ventricles with growth and diffusion of the tumor in the right lateral ventricle ( $\mathbf{C}$ and $\mathbf{F})$.

*The left ventricle looked normal.

Abbreviation: MRI, magnetic resonance imaging.

septostomy (Figure 6D), the neuroendoscopic exploration suggested normal features of the left ventricular system (Figure 6E). However, on closer examination, an initial contralateral tumor infiltration was evident through the fibers of the subependymal layer, situated along the anterior commissure (Figure 6F). This finding, undetected by preoperative neuroimaging studies (Figure 5D), became evident during the second postoperative MR image (Figure 5E). The postoperative course was uneventful, and her symptoms improved.

Histopathological examination showed a malignant epithelial neoplasm, organized in tubules and glands and occurring with aspects of mucosecretion, infiltrating the brain parenchyma. According to immunohistochemistry, the lesion was positive for cytokeratin 20 and CEA. The morphology of the lesion and the results of immunohistochemistry were consistent with metastasis from a gastrointestinal tumor (Figure 7). Postoperatively, further investigations to identify the primary lesion were performed (repeated total body computed tomography, MRI, and PET scans, repeated gastroscopy and colonoscopy, repeated computed tomographic colonography and colon capsule endoscopy), but the primary lesion remained unknown. The patient was treated with adjuvant radiotherapy and platinum-based chemotherapy (which is the standard protocol for unknown primary tumors at our institution). The patient survived for 20 months after surgery, with uncontrollable massive diffusion of the disease 2 months before death (Figure 5C and F).

\section{Discussion}

Intraventricular lesions are challenging due to their location deep inside the intracranial space and their proximity to critical neurovascular brain structures.

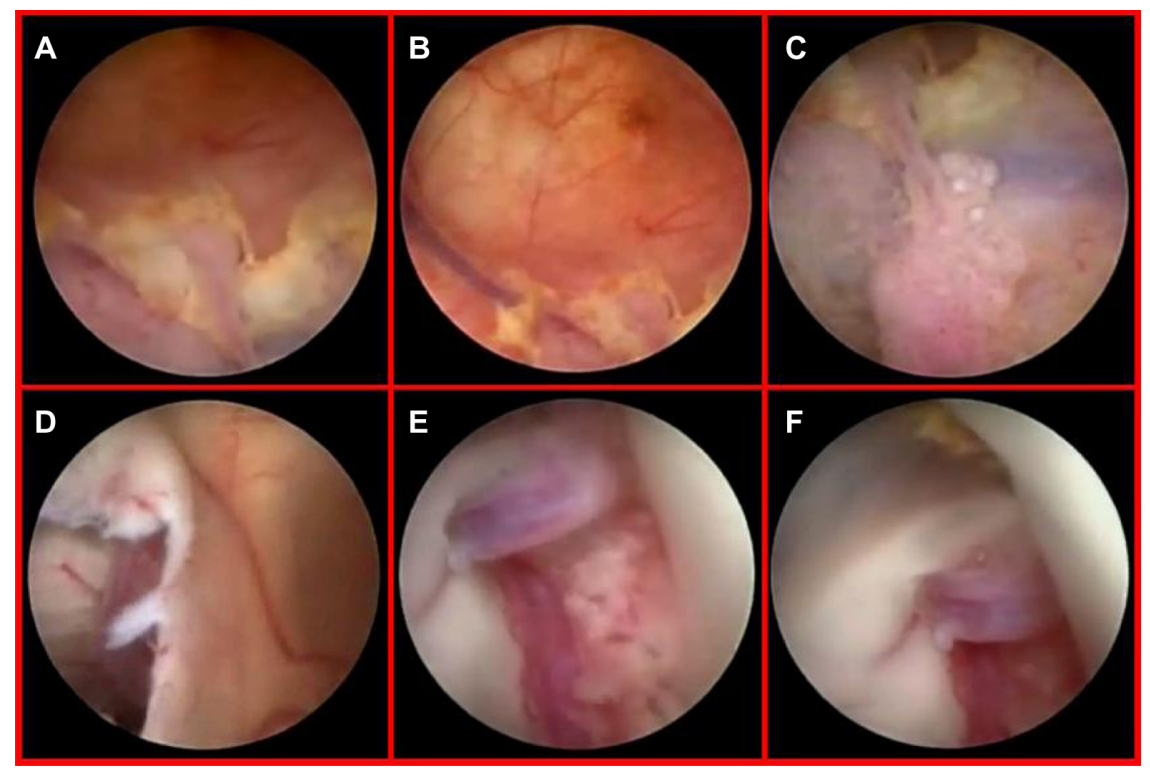

Figure 6 Diffuse yellowish coloration of the walls of the right lateral ventricle discovered during neuroendoscopic examination, with clusters of neoplastic cells embedding the choroid plexus and a thin sheet of tumor cells forming small nodules in the subependymal region (A-C). Endoscopic view of the septostomy with a contralateral apparently normal ependymal layer and choroid plexus ( $\mathbf{D}$ and $\mathbf{E}$ ). Close-up view of an initial, contralateral tumor infiltration evident through the fibers of the subependymal layer, situated along the anterior commissure (F). This finding was undetected by preoperative magnetic resonance imaging (see Figure 5D). 


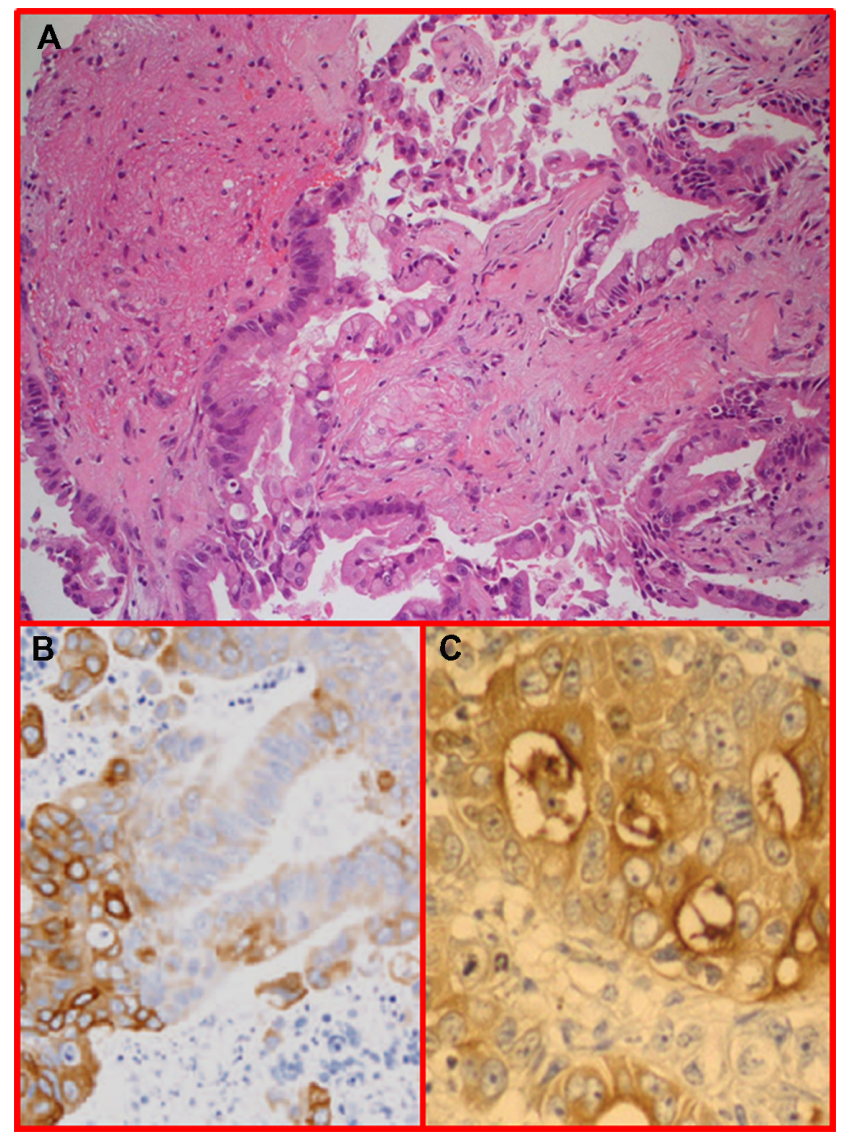

Figure $7 \mathrm{H} \& \mathrm{E}$ staining of neoplastic epithelial tubules and glands infiltrating the brain parenchyma.

Notes: The cells show aspects of mucosecretion (A). Immunohistochemistry was positive for cytokeratin 20 (B) and CEA (C).

Abbreviations: CEA, carcinoembryonic antigen; H\&E, hematoxiline and eosin.

These tumors, which usually have slowly progressive symptoms due to obstructive hydrocephalus, are ideal for the application of minimally invasive neuroendoscopic approaches because of their location inside a cavity filled with a transparent liquid. Compared with microsurgery, neuroendoscopy minimizes the risk related to dissection and brain retraction, providing clearer distinctions of the interface between the tumor and the surrounding eloquent structures. Consequently, neuroendoscopy permits the effective visualization of ependymal surfaces to diagnose unsuspected lesions and manages the associated hydrocephalus, restoring obstructed CSF pathways, via the same minimally invasive approach. Hence, in selected cases, endoscopic surgery may be as effective as microsurgery but less invasive, enabling a lower morbidity rate, a shorter operative time, and a shorter hospital stay for the patient. ${ }^{8,11-15}$

In the first case described, the patient had a recurrent GBM involving the third ventricle, while the right lateral ventricle, the initial operating site, apparently remained disease-free.
In the second case, the patient had metastatic intraventricular lesions intimately related to the choroid plexus of the right lateral ventricle, producing obstructive hydrocephalus by blocking CSF flow at the level of the foramen of Monro. In these cases, the neuroendoscopic approach represented a minimally invasive and effective treatment, permitting the acquisition of a histological diagnosis and enabling subsequent specific adjuvant therapy. The approach also controlled intracranial hypertension, relieving the CSF pathways by removal of strategic lesions, endoscopic ventriculocisternotomy of the third ventricle, and septostomy. Furthermore, in the first case, the neuroendoscopic approach allowed for the insertion of an intraventricular catheter connected to an Ommaya reservoir for intrathecal chemotherapy. ${ }^{16}$ Finally, neuroendoscopy allowed for an excellent visualization of ependymal surfaces infiltrated by small tumor nodules, revealing the subependymal dissemination of tumors below the threshold of detection by MRI.

To date, the mechanisms of the periventricular spreading of gliomas and metastases remain unknown, and the prognostic significance of these lesions remains unclear. Two hypotheses have been proposed. One is that the periventricular seeding of tumors can take place via the cerebrospinal pathway after ventricular system invasion, and deposit tumor cells in other parts of the ventricle and the subependymal and/or subpial regions. ${ }^{17,18}$ The choroid plexus may represent the portal of entry into the ventricular system for hematogenously disseminated pathogens, considering the relatively high cerebral blood flow in the choroid plexus (five times higher than brain parenchyma) and the fenestrated endothelium of its capillaries, through which solutes can pass. ${ }^{19,20}$ According to the other hypothesis, tumor dissemination can occur along fiber tracts and subependymal tissue as a diffuse sheet of advancing tumor cells. ${ }^{17}$ In fact, basic pathology studies have demonstrated (as described in Scherer's seminal studies ${ }^{21,22}$ over 50 years ago) and recent stereotactic biopsy studies have confirmed that glioma cells can invade the brain diffusely through the meningeal-subarachnoid space in a subependymal spread that migrates along cortical gray matter and white matter fibers. ${ }^{23-28}$ Moreover, experiments have confirmed that, especially during early stages of growth, glioma cells can migrate over long distances without necessarily requiring angiogenesis, taking advantage of preexistent host blood vessels from which they can spread in the subarachnoid space. Anti-angiogenic therapy, reducing the neovascularization in the area of tumor spread, can thus increase the other routes of invasion. ${ }^{29,30}$

Regarding GBM, authors who document tumor dissemination raise important questions about the biological 
behaviors of glioma, its clinical and pathological presentation, and the role of therapies in preventing recurrence. ${ }^{3,31}$ In recent years, the region adjacent to the lateral ventricles has been an area of increasing interest as a potential source for studies of malignant tumors and other aggressive diseases. Basic studies have demonstrated that the human brain's subventricular zone (SVZ), which is located just under the ependyma of the brain ventricles, harbors astrocyte-like neural cells, which possess the capacity of stem cells to undergo self-renewal and multilineage differentiation. ${ }^{32}$ Interestingly, there is also a subpopulation of cells within brain tumors that possess these same characteristics. ${ }^{33-36}$ Moreover, in animal models, gliomas, including GBM, can be induced from SVZ cells. ${ }^{37-39}$ Based on these findings, the emerging hypothesis is that brain tumor stem cells may be derived from neural stem cells and that both of these populations may originate in the SVZ. ${ }^{40,41}$

The SVZ may also provide an environmental niche that supports tumorigenesis. In fact, the SVC is rich in extracellular matrix proteins, including basal lamina, ${ }^{42,43}$ tenascin-C and chondroitin sulfate, ${ }^{44-46}$ as well as microglia and endothelial cells. ${ }^{47}$ All these proteins play an important role in tumor progression and migration. Hence, tumors that occur in this region may have different features than others that occur in different anatomical regions. ${ }^{40,48}$ In their study, Lim et $\mathrm{al}^{49}$ analyzed MRI features of GBMs in specific relation to the SVZ. They observed that GBMs contacting the SVZ were most likely to be multifocal at the time of initial diagnosis and that GBMs recurred at great distances from the initial site of the lesion(s). Conversely, GBMs not involving either the SVZ or the cortex occurred only as solitary lesions, and their recurrences were always contiguous with the resection cavity. ${ }^{49}$ Moreover, it has been reported that, among patients who present with disseminated GBMs, the patients with tumors with a subependymal spread have a worse prognosis. ${ }^{3}$ Chaichana et al ${ }^{50}$ reported that GBMs adjacent to the lateral ventricles may lead to poorer patient outcomes, depending on the GBMs' anatomical localization close to critical neurological brain structures (which could increase the morbidity rate) and on the GBMs' particular cellular environment (which could make them more invasive and infiltrative). ${ }^{50}$ Thus, for patients with GBMs contacting the SVZ, a radiation therapy that includes locations distant from the SVZ may be needed. ${ }^{49}$

Few authors have analyzed the mechanisms underlying the periventricular dissemination of brain metastases, probably because intraventricular metastases are rare. Although the exact number of incidents is difficult to determine, best estimates suggest that they comprise about $6 \%$ of all intraventricular tumors. ${ }^{51}$ Patients with intraventricular metastases may or may not be known to have a systemic cancer at the time of presentation. In patients with known systemic cancer, an intraventricular mass showing postcontrast enhancement on neuroradiological imaging must be considered as a metastasis until proven otherwise. ${ }^{2}$ In patients without known systemic cancer, as in the presented case, the differential diagnosis of an intraventricular lesion is much more indeterminate. According to some authors, the finding of an extensive perilesional edema is more characteristic of metastases than other intraventricular lesions. ${ }^{51-54}$ However, other authors have reported that intraventricular metastases can mimic meningiomas or colloid cysts. ${ }^{55,56}$

Choroid plexus metastasis accounts for approximately $0.14 \%$ of patients with cerebral metastases and is a diagnosis invariably associated with a poor prognosis. ${ }^{20}$ It can develop hematogenously, through the anterior or posterior choroidal artery, or it can spread through the CSF. ${ }^{51}$ In the described case, we considered that the tumor cells invaded the ventricular system through the choroid plexus of the lateral ventricle, followed by extensive, diffuse subependymal periventricular dissemination. The choroid plexus of the lateral ventricle, as mentioned above, may represent the "entrance" to the ventricular system for hematogenously disseminated pathogens, considering its relatively high cerebral blood flow and the lack of an intact blood-brain barrier, two major determinants of cerebral metastasis. ${ }^{17,20}$ Nevertheless, choroid plexus metastases have a very low incidence.

According to the "seed and soil" hypothesis, the organ distribution of metastases produced by different human neoplasms is not due to chance but, rather, certain tumor cells (the "seed") that have a specific affinity for the milieu of certain organs (the "soil"). Metastases result only when the seed and soil are compatible. ${ }^{57}$ Therefore, a potential explanation for the scarcity of choroid plexus metastasis may be the inhospitable "soil" of the choroid plexus. In fact, the choroid plexus has features that are different from the rest of the brain microvasculature (it readily accumulates immune complexes targeted by anti-basement membrane antibodies, it has a tendency to accumulate amyloid, and it has a collagenous stroma) and may prevent tumor cell implantation. ${ }^{58}$ Moreover, the high blood flow may block the metastatic process, opposing the blood-borne tumor cells' extravasation and the seeding of the plexus stroma. ${ }^{20}$ Finally, the lack of specific tumor-cell receptors in the choroid plexus microenvironment may be a third potential inhospitable factor. ${ }^{20,59}$ Thus, biologic factors could play a major role in determining the occurrence 
of intraventricular metastases, as suggested by the low rate of metastases from lung cancer (the most common systemic tumor) compared with the high rate of metastases from renal and gastrointestinal primaries. ${ }^{2,20}$

\section{Conclusion}

In the presented cases, neuroendoscopy allowed for the targeted removal of strategic intraventricular lesions via a minimally invasive approach, permitting the acquisition of a histological diagnosis and the control of intracranial hypertension by treating the associated hydrocephalus. Neuroendoscopy has also enabled us to identify the subependymal spread of a tumor below the threshold of detection by MRI. Moreover, it has enabled us to define the tumor's characteristics and arrangement, and to speculate about the mechanisms underlying the tumor's diffusion route and its prognostic significance. Thanks to the neuroendoscopic approach, we acquired important data that might play a leading role in defining optimally tailored management protocols for GBM and brain metastases.

\section{Disclosure}

The authors report no conflicts of interest in this work.

\section{References}

1. Ribalta T, Fuller GN. Brain Tumors: An Overview of Histopathologic Classification. In: Winn HR, editor. Youman's Neurosurgical Surgery. 5th ed. Philadelphia: Saunders; 2003:661-672.

2. Vecil GG, Lang FF. Surgical resection of metastatic intraventricular tumors. Neurosurg Clin N Am. 2003;14(4):593-606.

3. Parsa AT, Wachhorst S, Lamborn KR, et al. Prognostic significance of intracranial dissemination of glioblastoma multiforme in adults. J Neurosurg. 2005;102(4):622-628.

4. Li J, Wang M, Won M, et al. Validation and simplification of the Radiation Therapy Oncology Group recursive partitioning analysis classification for glioblastoma. Int J Radiat Oncol Biol Phys. 2011;81(3): 623-630

5. Hartmann C, Meyer J, Balss J, et al. Type and frequency of IDH1 and IDH2 mutations are related to astrocytic and oligodendroglial differentiation and age: a study of 1,010 diffuse gliomas. Acta Neuropathol. 2009;118(4):469-474.

6. Capper D, Weissert S, Balss J, et al. Characterization of R132H mutation-specific IDH1 antibody binding in brain tumors. Brain Pathol. 2010;20(1):245-254.

7. Combs SE, Rieken S, Wick W, et al. Prognostic significance of IDH-1 and MGMT in patients with glioblastoma: one step forward, and one step back? Radiat Oncol. 2011;6:115.

8. Cappabianca P, Cinalli G, Gangemi M, et al. Application of neuroendoscopy to intraventricular lesions. Neurosurgery. 2008;62(2): SHC575-SHC598.

9. Gerstner ER, Yip S, Wang DL, Louis DN, Iafrate AJ, Batchelor TT. MGMT methylation is a prognostic biomarker in elderly patients with newly diagnosed glioblastoma. Neurology. 2009;73(18): 1509-1510.

10. Stupp R, Mason WP, van den Bent MJ, et al. Radiotherapy plus concomitant and adjuvant temozolomide for glioblastoma. $N$ Engl J Med. 2005;352(10):987-996.

11. Gaab MR, Schroeder HW. Neuroendoscopic approach to intraventricular lesions. J Neurosurg. 1998;88(3):496-505.
12. Yasargil MG, Abdulrauf SI. Surgery of intraventricular tumors. Neurosurgery. 2008;62(6):SHC1029-SHC1041.

13. Hayashi N, Murai H, Ishihara S, et al. Nationwide investigation of the current status of therapeutic neuroendoscopy for ventricular and paraventricular tumors in Japan. $J$ Neurosurg. 2011;115(6): $1147-1157$.

14. Chowdhry SA, Cohen AR. Intraventricular neuroendoscopy: complication avoidance and management. World Neurosurg. 2012. Available from: http://www.worldneurosurgery.org/article/S18788750(12)00163-5. Accessed August 27, 2012.

15. Oi S, Kamio M, Joki T, Abe T. Neuroendoscopic anatomy and surgery in pineal region tumors: role of neuroendoscopic procedure in the "minimally-invasive preferential" management. J Neurooncol. 2001; 54(3):277-286.

16. Iacoangeli M, Roselli R, Pagano L, et al. Intrathecal chemotherapy for treatment of overt meningeal leukemia: comparison between intraventricular and traditional intralumbar route. Ann Oncol. 1995;6(4): 377-382.

17. McGeachie RE, Gold LH, Latchaw RE. Periventricular spread of tumor demonstrated by computed tomography. Radiology. 1977;125(2): $407-410$.

18. Vannier A, Gray F, Gherardi R, Marsault C, Degos JD, Poirier J. Diffuse subependymal periventricular metastases: report of three cases. Cancer. 1986;58(12):2720-2725.

19. Levine S. Choroid plexus: target for systemic disease and pathway to the brain. Lab Invest. 1987;56(3):231-233.

20. Al-Anazi A, Shannon P, Guha A. Solitary metastasis to the choroid plexus. Case illustration. J Neurosurg. 2000;92(3):506.

21. Scherer HJ. Structural development in gliomas. Am J Cancer. 1938;34: 333-351.

22. Scherer HJ. The forms of growth in gliomas and their practical significance. Brain. 1940;63(1):1-35.

23. Daumas-Duport C, Scheithauer BW, Kelly PJ. A histologic and cytologic method for the spatial definition of gliomas. Mayo Clin Proc. 1987;62(6):435-449.

24. Laws ER, Goldberg WJ, Bernstein JJ. Migration of human malignant astrocytoma cells in the mammalian brain: Scherer revisited. Int J Dev Neurosci. 1993;11(5):691-697.

25. Bernstein JJ, Goldberg WJ, Laws ER. Human malignant astrocytoma xenografts migrate in rat brain: a model for central nervous system cancer research. J Neurosci Res. 1989;22(2):134-143.

26. Tamura M, Ohye C, Nakazato Y. Pathological anatomy of autopsy brain with malignant glioma. Neurol Med Chir (Tokyo). 1993;33(2): $77-80$.

27. Kato K, Yoshida J, Kageyama N. Clinico-pathological studies of CSF dissemination of glioblastoma and medulloblastoma. No Shinkei Geka. 1986;14(8):981-987. Japanese.

28. Nishio S, Korosue K, Tateishi J, Fukui M, Kitamura K. Ventricular and subarachnoid seeding of intracranial tumors of neuroectodermal origin - a study of 26 consecutive autopsy cases with reference to focal ependymal defect. Clin Neuropathol. 1982;1(2):83-91.

29. Guillamo JS, Lisovoski F, Christov C, et al. Migration pathways of human glioblastoma cells xenografted into the immunosuppressed rat brain. J Neurooncol. 2001;52(3):205-215.

30. Lamszus K, Kunkel P, Westphal M. Invasion as limitation to antiangiogenic glioma therapy. Acta Neurochir Suppl. 2003;88:169-177.

31. Iacoangeli M, Roselli R, Prezioso A, Scerrati M, Rossi GF. Staging of supratentorial hemispheric glioma using tumour extension, histopathological grade and extent of surgical resection. Br J Surg. 1993;80(9): $1130-1133$.

32. Sanai N, Tramontin AD, Quinones-Hinojosa A, et al. Unique astrocyte ribbon in adult human brain contains neural stem cells but lacks chain migration. Nature. 2004;427(6976):740-744.

33. Ignatova TN, Kukekov VG, Laywell ED, Suslov ON, Vrionis FD, Steindler DA. Human cortical glial tumors contain neural stemlike cells expressing astroglial and neuronal markers in vitro. Glia. 2002;39(3):193-206. 
34. Hemmati HD, Nakano I, Lazareff JA, et al. Cancerous stem cells can arise from pediatric brain tumors. Proc Natl Acad Sci U S A. 2003; 100(25):15178-15183.

35. Galli R, Binda E, Orfanelli U, et al. Isolation and characterization of tumorigenic, stem-like neural precursors from human glioblastoma. Cancer Res. 2004;64(19):7011-7021.

36. Singh SK, Hawkins C, Clarke ID, et al. Identification of human brain tumour initiating cells. Nature. 2004;432(7015):396-401.

37. Zhu Y, Guignard F, Zhao D, et al. Early inactivation of p53 tumor suppressor gene cooperating with NF1 loss induces malignant astrocytoma. Cancer Cell. 2005;8(2):119-130.

38. Holland EC, Celestino J, Dai C, Schaefer L, Sawaya RE, Fuller GN. Combined activation of Ras and Akt in neural progenitors induces glioblastoma formation in mice. Nat Genet. 2000;25(1):55-57.

39. Savarese TM, Jang T, Low HP, et al. Isolation of immortalized, INK4a/ ARF-deficient cells from the subventricular zone after in utero N-ethylN-nitrosourea exposure. J Neurosurg. 2005;102(1):98-108.

40. Sanai N, Alvarez-Buylla A, Berger MS. Neural stem cells and the origin of gliomas. N Engl J Med. 2005;353(8):811-822.

41. Vescovi AL, Galli R, Reynolds BA. Brain tumour stem cells. Nat Rev Cancer. 2006;6(6):425-436.

42. Alvarez-Buylla A, Lim DA. For the long run: maintaining germinal niches in the adult brain. Neuron. 2004;41(5):683-686.

43. Ljubimova JY, Fujita M, Khazenzon NM, Ljubimov AV, Black KL. Changes in laminin isoforms associated with brain tumor invasion and angiogenesis. Front Biosci. 2006;11(1):81-88.

44. Chekenya M, Rooprai HK, Davies D, Levine JM, Butt AM, Pilkington GJ. The NG2 chondroitin sulfate proteoglycan: role in malignant progression of human brain tumours. Int J Dev Neurosci. 1999;17(5):421-435.

45. Orend G, Chiquet-Ehrismann R. Tenascin-C induced signaling in cancer. Cancer Lett. 2006;244(2):143-163.

46. Wang D, Anderson JC, Gladson CL. The role of the extracellular matrix in angiogenesis in malignant glioma tumors. Brain Pathol. 2006;15(4):318-326.

47. Calabrese C, Poppleton H, Kocak M, et al. A perivascular niche for brain tumor stem cells. Cancer Cell. 2007;11(1):69-82.
48. Quinones-Hinojosa A, Chaichana K. The human subventricular zone: a source of new cells and a potential source of brain tumors. Exp Neurol. 2007;205(2):313-324.

49. Lim DA, Cha S, Mayo MC, et al. Relationship of glioblastoma multiforme to neural stem cell regions predicts invasive and multifocal tumor phenotype. Neurooncol. 2007;9(4):424-429.

50. Chaichana K, Parker S, Olivi A, Quiñones-Hinojosa A. A proposed classification system that projects outcomes based on preoperative variables for adult patients with glioblastoma multiforme. J Neurosurg. 2010;112(5):997-1004.

51. Kohno M, Matsutani M, Sasaki T, Takakura K. Solitary metastasis to the choroid plexus of the lateral ventricle: report of three cases and a review of the literature. J Neurooncol. 1996;27(1):47-52.

52. Kadrian D, Tan L. Single choroid plexus metastasis 16 years after nephrectomy for renal cell carcinoma: case report and review of the literature. J Clin Neurosci. 2004;11(1):88-91.

53. Tanimoto M, Tatsumi S, Tominaga S, et al. Choroid plexus metastasis of lung carcinoma - case report. Neurol Med Chir (Tokyo). 1991;31(3): $152-155$.

54. Mizuno M, Asakura K, Nakajima S, et al. Renal cell carcinoma metastasizing to choroid plexus of lateral ventricle: a case report. No Shinkei Geka. 1992;20(4):469-474. Japanese.

55. Killebrew K, Krigman M, Mahaley MS, Scatliff JH. Metastatic renal cell carcinoma mimicking a meningioma. Neurosurgery. 1983;13(4): $430-434$.

56. Leach JC, Garrott H, King JA, Kaye AH. Solitary metastasis to the choroid plexus of the third ventricle mimicking a colloid cyst: a report of two cases. J Clin Neurosci. 2004;11(5):521-523.

57. Paget $\mathrm{S}$. The distribution of secondary growths in cancer of the breast. 1889. Cancer Metastasis Rev. 1989;8(2):98-101.

58. McIntosh RM, Copack P, Chernack WB, Griswold WR, Weil R 3rd, Koss MN. The human choroid plexus and autoimmune nephritis. Arch Pathol. 1975;99(1):48-50.

59. Fidler IJ. The role of the organ microenvironment in brain metastasis. Semin Cancer Biol. 2011;21(2):107-112.
OncoTargets and Therapy

\section{Publish your work in this journal}

OncoTargets and Therapy is an international, peer-reviewed, open access journal focusing on the pathological basis of all cancers, potential targets for therapy and treatment protocols employed to improve the management of cancer patients. The journal also focuses on the impact of management programs and new therapeutic agents and protocols on

\section{Dovepress}

patient perspectives such as quality of life, adherence and satisfaction The manuscript management system is completely online and includes a very quick and fair peer-review system, which is all easy to use. Visit http://www.dovepress.com/testimonials.php to read real quotes from published authors. 